\title{
Rol de las redes sociales en el desarrollo físico y mental de los adultos mayores
}

\author{
Recibido: 10 de agosto de 2018 \\ Aceptado: 9 de octubre de 2018 \\ Publicado: 28 de noviembre de 2018
}

\author{
Moncerrat Arango Morales \\ monserrat.arangom@uanl.mx \\ Lucinda Sepúlveda García \\ lucinda.sepulvedag@uanl.mx \\ Hiram Garrido Ledezma \\ hiram.garridold@uanl.edu.mx
}

Universidad Autónoma de Nuevo León (México)

Resumen: La presente investigación tiene como objetivo analizar si los adultos mayores de los municipios de Monterrey y Apodaca en el Estado de Nuevo León (México) cuentan con herramientas electrónicas que le permitan el acceso y uso de redes sociales, y detallar la interacción que tienen con pares, familiares e instituciones con las que tengan alguna relación, como alternativa de integración. Se utilizó un método mixto para la recolección de los datos, una muestra de 435 sujetos a fin de garantizar la representatividad. De la investigación resulta que el $49.4 \%$ (215) de adultos mayores expone el sentimiento de alegría como el código más repetido porque pueden comunicarse con sus familiares amigos y conocidos, obtiene información diversa y les facilita tareas y trabajos. Los adultos mayores consumen la red Facebook. Además, se concluye que los adultos mayores perciben las redes sociales como parte integral de su ser con sentido de pertenencia e inclusión social.

Palabras clave: Adulto mayor, redes sociales, brecha digital, integración, inclusión.

\begin{abstract}
This research aims to analyze whether the adults in the municipalities of Monterrey and Apodaca in the state of Nuevo León (Mexico) have electronic tools that allow access and use of social networks, and detailing interaction they have with peers, family members, and institutions that have some connection, as an alternative to integration. It was used a mixed method for data collection, a sample of 435 subjects in order to ensure representativeness. From this research, it turns out that the 49.4\% (215) of older adults exposes the feelings of joy as the most repeated code because they can communicate with their relatives, friends
\end{abstract}


and acquaintances, get diverse information and facilitates their tasks and work. Older adults should consume Facebook. Moreover, it is concluded that seniors perceive social networks as an integral part of their being with a sense of belonging and social inclusion.

Key words: Older Adult, Social Networks, Digital Gap, Integration, Inclusion.

\section{Introducción}

México es un país de niños y jóvenes (INEGI, 2014), pero esto no siempre ha sido así. En los setentas ocurrieron una serie de cambios como consecuencia del aumento de las tasas de natalidad. De esta manera, la estructura de las edades se ha modificado. Actualmente, se prevé que la proporción de personas mayores de 60 años aumente. Este rango de edad es reconocido por organizaciones como el Instituto Nacional de las Personas Adultas Mayores (INAPAM) y la Secretaría de Salud. Sin embargo, el Instituto Nacional de la Mujeres (Inmujeres, 2015) señala que este proceso de deterioro depende de signos que determinan el envejecimiento como la edad física, psicológica y social, así como su historia, cultura y condiciones socioeconómicas de las personas, siendo de carácter individual.

La presente investigación versa sobre las redes sociales y su relación con los adultos mayores, por lo que es importante entender cuáles son los indicadores fundamentales para entender esta vinculación. Sobre esto, el INEGI (2014) señala que el porcentaje de individuos alfabetizados ha cambiado: de un $69.7 \%$, en 2000 , se ha incrementado en un $74.3 \%$, en el 2010 .

El enfoque se centra en dos municipios de Nuevo León, considerado como el Estado industrial de México, Monterrey es una ciudad económicamente importante para el desarrollo de la nación mexicana y Apodaca es un municipio que lleva varios años experimentando un importante crecimiento urbano y poblacional, donde además se asientan grandes industrias, fuente de varios puestos de trabajos.

Según datos de la Encuesta Intercensal 2015 (INEGI, 2015), de 1706378 ciudadanos que conforman los municipios de Monterrey y Apodaca Nuevo León, 597207 habitan en el municipio de Apodaca y de ellos, 27865 son adultos mayores. En tanto que en el Municipio de Monterrey se contaba con una población de 1109171 y de ellos 158570 son adultos mayores (tabla 1). Este grupo poblacional (conformado por un total de 186 435) tiene una edad que supera los 60 años, donde se destaca la suma de todas las experiencias de vida y pasa por la mayoría de las metas familiares, profesionales y sociales. Es en esta etapa donde, tanto hombres como mujeres, presentan condiciones de vulnerabilidad física, social y económica (Pensionissste, 2017). 
Tabla 1: Población por edades a partir de 60 años de los municipios de Monterrey y Apodaca de Nuevo León.

\begin{tabular}{|c|c|c|}
\hline POBLACIÓN & MONTERREY & APODACA \\
\hline Población total & 2331030 & 213990 \\
\hline $60-64$ & 89466 & 3696 \\
\hline $65-69$ & 77883 & 2286 \\
\hline $70-74$ & 57408 & 1602 \\
\hline $75-79$ & 37596 & 990 \\
\hline $80-84$ & 24375 & 414 \\
\hline $85-89$ & 13338 & 234 \\
\hline $90-94$ & 4641 & 96 \\
\hline 95-99+ & 1053 & 36 \\
\hline $\begin{array}{l}\text { Población total } \\
\text { adultos mayores }\end{array}$ & 305760 & 9354 \\
\hline
\end{tabular}

Fuente: Elaboración propia, basada en INEGI (2015).

Esta investigación tiene como objetivo analizar los tipos de dispositivos empleados, los usos de las redes sociales, determinar el comportamiento y los sentimientos que les proporciona esos recursos; si dichas herramientas funcionan y facilitan su vida diaria, así como recoger sus puntos de vista sobre las redes sociales y la Internet. Recordemos que este segmento son la generación que no creció con estas herramientas tecnológicas (como el caso de los millennials), por tanto, los resultados de este trabajo de investigación contribuirá a dignificar este sector de la población y acortar las diferencias en lo referente al uso de las herramientas digitales.

Sobre este grupo en particular, la esperanza de vida ha cambiado. Esto puede considerarse un éxito de las políticas públicas en salud pública y el desarrollo socioeconómico del país. Empero, esto también representa un reto para la sociedad, ya que los adultos mayores deben ser considerados no solo en términos de salud, sino también como individuos importantes en lo referente a su participación social y seguridad.

Como parte de esta participación, los gobiernos (en sus diferentes niveles) deben poner atención a la integración de los adultos mayores en las cuestiones tecnológicas. La brecha generacional debe ser acortado, sobre todo si se toma en cuenta que de los 60 a los 69 años, el $44.3 \%$ de esta comunidad se encuentra activa; en tanto que el $34.3 \%$ realiza actividades domésticas y el 16.2\% se encuentran pensionados o jubilados (INEGI , 2015, p. 13). 


\section{Adultos mayores y la brecha digital}

Las sociedades se encuentran mezcladas por ciudadanos de diversas edades. Sin embargo, el aumento poco equilibrado y el crecimiento continuo de personas mayores de 60 años va a provocar que México se convierta en un país cada vez más envejecido, lo cual plantea un reto para incluir (de manera continua y sistemática) a este grupo vulnerable, proporcionándoles mayor bienestar e integración no solo en el orden cultural, sino también a través de programas sociales que les permitan la educación en la tecnologías digitales.

Cerda (2005) señala que la edad es una razón importante en la distancia que se produce entre las generaciones, ya que las realidades y experiencias de vida son totalmente distintas. Sin embargo, ambas generaciones se encuentran en esta época en la que convergen aunque con diferentes significados para cada uno de los grupos, ya que tienen experiencias y construcciones simbólicas distintas. Esto puede ser la causa de que ambas generaciones sientan que viven en mundos diferentes, porque sus competencias son distintas, quedando los adultos mayores distantes de las nuevas enseñanzas tecnológicas.

Los adultos mayores ni nacieron ni crecieron a la par de la web, ya que esta tiene aproximadamente 23 años de vida. Términos como "e-mail", "Facebook", "tablet" o "smartphone" pueden llegar a ser complicados de entender para este segmento. Esto no significa que deban quedar excluidos del uso de estas tecnologías que, en la actualidad, pueden ayudar a mejorar su calidad de vida en diferentes aspectos. Morris y Branding, citados por Vilte, Martin, Gaetan y Saldaño (2014), demuestran que el correo electrónico es un factor importante en sus procesos de comunicación, ya que les permite contactarse con todos sus familiares, muchos de ellos ubicados en diversas partes del país y del mundo. Así, la web se presenta como la herramienta que les permite aminorar distancias.

En estos requerimientos tecnológicos se produce la brecha digital y los adultos mayores quedan al margen de los nuevos aprendizajes, convirtiéndose en "analfabetos digitales", porque no cuentan con los conocimientos necesarios para manejar y usar las nuevas tecnologías (Cerda, 2005), debilitándolos para enfrentar el mundo actual, cada vez más dependiente de las herramientas tecnológicas para cubrir necesidades sociales, culturales, políticas y, por supuesto, de entretenimiento y diversión.

Las sociedades se encuentran inmersas en el uso de las nuevas tecnologías, por lo que es necesario que estas herramientas se encuentren a la mano de todos los ciudadanos independientemente de la edad y los adultos mayores no deben ser la excepción: ellos deben familiarizarse con estos recursos mediante estrategias que provoquen la apropiación y el dominio de las tecnologías. En ese sentido, es necesario que ingresen a un proceso educativo para manejar estas herramientas, no solamente por cuestiones sociales, sino también para acceder a información política necesaria para su participación ciudadana en todo evento de elección popular.

Uno de los estereotipos relacionados con las tecnologías de la información dictamina que estos son diseñados para la "gente joven"; no obstante, es inexistente el principio que señale que Internet esté reservado a personas de algún determinado rango de edad (Barroso, 
Cabero \& Romero, 2002). Inmujeres (2015) prevé que para 2050 "las mujeres de 60 años y más representen $23.3 \%$ del total de población femenina y los hombres constituyan $19.5 \%$ del total de la masculina" (p. 5), el 42.8\% de la población total serán individuos mayores a 60 años de edad, a los que deberá procurarse una mejor calidad de vida, a fin de que gocen su proceso de envejecimiento de forma plena y sirviendo de instrumentos esenciales para su participación cívica (Culver \& Jacobson, 2012).

Asimismo, se habla de una "fobia" de la población de este estudio hacia las tecnologías de la información y la comunicación (Montaña, Estanyol \& Lalueza, 2015). En todo caso, la actitud de este segmento ante estas herramientas tecnológicas puede mostrarse receptiva cuando el entorno es apto y se aplica la enseñanza de una manera adecuada (Kiel, 2005). Visto de esta manera, el término "brecha digital" se presenta como importante, ya que cuantifica la diferencia existente entre países, sectores y personas que tienen acceso a los instrumentos y herramientas de información y la capacidad de utilizarlos (Agudo, Pascual \& Fombona, 2012), configurándose como

el desfase o división entre individuos, hogares, áreas económicas y geográficas con diferentes niveles socioeconómicos con relación tanto en sus oportunidades de acceso a las tecnologías de la información y la comunicación (TIC), como al uso de internet para una amplia variedad de actividades (OCDE, 2011, p. 5).

La brecha digital no es únicamente la falta de acceso a Internet ("primera brecha digital"), sino también comprende los usos y la intensidad del uso de la Internet ("segunda brecha digital"), donde se toman en cuenta conceptos como la alfabetización digital, competencias digitales o la inclusión digital (Abad, 2014). Por ese motivo, debe tomarse en cuenta data tanto de la brecha como de la inclusión digital, entendida como "el conjunto de políticas públicas relacionadas con la construcción, administración, expansión, ofrecimiento de contenidos y desarrollo de capacidades locales en las redes digitales públicas, alámbricas e inalámbricas, en cada país y en la región entera" (Cumbre Mundial sobre la Sociedad de la Información, 2004).

El uso de la Internet está muy relacionado con características sociodemográficas de los propios usuarios, tales como la edad, nivel de estudio, clase social y situación laboral (Robles \& Molina, 2007), de tal manera que son las personas más jóvenes las que mayoritariamente usan la web y el porcentaje de usuarios disminuye conforme la edad de los usuarios avanza. En todo caso, el uso de las TIC brindaría a los adultos mayores el acceso a una mayor cantidad de información y comunicación, así como a programas educativos y a fuentes de generación de recursos. Tener conocimiento del uso de estas herramientas les daría autonomía en su vida social y la oportunidad de desarrollar su potencial, viviendo con seguridad y dignidad (Padilla \& Padilla, 2008).

\section{Educación digital}

Colombo, Aroldi \& Carlo (2015) señalan que el uso de las TIC por los adultos mayores está supeditado al contexto socioeconómico, de tal manera que quienes tengan poder 
adquisitivo contarán con un capital social y cultural más grande. El peligro en este caso es que dicha polarización socioeconómica puede hacer que las clases más humildes continúen marginados y desconectados de las nuevas tendencias en cuanto a las actuales herramientas de comunicación a nivel global.

Al llegar a la vejez, los adultos mayores se desvinculan de diversos espacios donde establecieron relaciones de diversa índole y en las que invertían una parte importante de su tiempo. Algunas de estas desvinculaciones son consecuencia de la terminación de su vida productiva en el mercado laboral, culminación de su rol reproductivo y fallecimiento de pares (Cardozo, Martín \& Saldaño, 2017). Este sector de la población es consciente de que las novedades tecnológicas les generan incertidumbre porque alteran sus hábitos, costumbres y estructuras mentales, aunque reconocen que pueden adaptarse mediante un aprendizaje continuo (Pavón, 2000). Desde el punto de vista político, este segmento se configura como un importante capital humano para la sociedad.

Agudo, Pascual y Fombona (2012) identifican cuatro tipos de uso de la Internet: formación, información, comunicación y entretenimiento, desprendiéndose que los individuos que tienen un nivel de educación superior (universitario) son los que disfrutan más su uso. Los adultos mayores señalan que lo utilizan para obtener un poco de entretenimiento y como hobby, pero que no lo necesita. Esta falta de necesidad puede ser consecuencia del miedo y porque la consideran un mundo demasiado complejo por los altos costos. Sin embargo, cuando los adultos mayores llegan a dominar esta tecnología, disfrutan de un bienestar psicológico, obteniendo una mejor posición frente a su familia e incluso ante la sociedad (Cardozo, Martín \& Saldaño, 2017).

\section{Metodología}

Para el desarrollo de esta investigación, se realizó un estudio mixto, exploratorio transversal, con el objetivo de determinar si los adultos mayores determinan la interacción a través de las herramientas que aplican en las redes sociales, haciendo énfasis en el uso y bienestar de las necesidades individuales para la inclusión social. Es exploratorio porque se aproxima a la relación que tienen los adultos mayores con las redes sociales y la Internet. Asimismo, es transversal porque el trabajo de campo se desarrolló en un tiempo específico (agostooctubre de 2017).

Se utiliza el sistema SPSS para el análisis de los datos, siendo la forma de medir la confiabilidad del instrumento a través de análisis no paramétricos. Mediante la aplicación de dicho sistema, se aplica la prueba de chi-cuadrado, que traza comparaciones entre las frecuencias observadas empíricas y se podrá generar un nivel de significación asintótica.

La muestra seleccionada (de tipo aleatorio estratificada) fue de 185 adultos mayores de 60 años en adelante. Se clasificó a los sujetos a través de diversas variables (edad, género, uso de redes sociales, entre otros). 


\section{Resultados}

A partir del análisis del sistema SPSS sobre los 435 cuestionarios (aplicándose 16 reactivos), muestran que el 57\% (250) son de Monterrey y 42.5\% (185) de Apodaca. Los rangos de edades que destacan son los de 60-65 años $(41.8 \%, 182), 66-70$ años $(31 \%, 135)$ y $71-75$ años $(14.9 \%, 65)$. De este grupo, el $63.2 \%$ (275) son mujeres y el $36.8 \%(160)$ son hombres.

El 83.2\% (362) se encuentra en posesión de un dispositivo electrónico, mientras que el $16.8 \%$ (73) no cuenta con ese recurso. El 74.7\% (325) goza de acceso a la Internet, en tanto que el $24.4 \%$ (106) está offline. La herramienta más utilizada para conectarse con el ciberespacio es el teléfono $75.6 \%$ (329), seguido de la computadora de escritorio (22.3\%, $97)$, la computadora portátil $(21.8 \%, 95)$ y la tablet $(20 \%, 87)$.

Con referencia a la frecuencia del uso de dichos equipos electrónicos, el 44.1\% (193) los utiliza diariamente; el 18.2\% (79) varias veces a la semana; el 16.6\% (72) de forma ocasional y varias veces al mes solamente el $3.7 \%$ (16). Como se observa en la tabla 2, Facebook se presenta como la red social más visitada $(40.9 \%, 178)$, seguido por el WhatsApp $(25.5 \%, 111)$; YouTube $(6.7 \%, 29)$ y Skype $(0.7 \%, 3)$. Un número importante de individuos no contestaron $(22.1 \%, 96)$, lo cual revela que no utilizan redes sociales, sino solamente Internet.

Tabla 2: ¿Cuál es la red social que más utiliza?

\begin{tabular}{|c|c|c|c|}
\hline \multicolumn{1}{|c|}{} & FRECUENCIA & PORCENTAJE \\
\hline \multirow{4}{*}{ Facebook } & 178 & 40.9 \\
\cline { 2 - 4 } & WhatsApp & 111 & 25.5 \\
\cline { 2 - 4 } & YouTube & 29 & 6.7 \\
\cline { 2 - 4 } & Skype & 3 & .7 \\
\cline { 2 - 4 } & Twitter & 2 & .5 \\
\cline { 2 - 4 } & Instagram & 1 & 3.4 \\
\cline { 2 - 4 } & Otra & 15 & 22.1 \\
\cline { 2 - 4 } & No contestó & 96 & $\mathbf{1 0 0 . 0}$ \\
\cline { 2 - 4 } & TOTAL & $\mathbf{4 3 5}$ & .2 \\
\hline
\end{tabular}

Fuente: Elaboración propia. 
Los encuestados invierten una hora para navegar $(30.1 \%, 131)$, dos horas $(16.6 \%, 72)$; tres horas $(16.3 \%, 71)$, cuatro horas $(8.3 \%, 36)$ y más de cuatro horas $(7.4 \%, 32)$. La tabla 3 señala que establecer conversaciones con amigos y familiares es la principal razón para usar la computadora $(30.8 \%, 134)$, seguido de correos electrónicos $(16.6 \%, 72)$, redes sociales $(14.0 \%, 61)$ y búsqueda de información $(8.3 \%, 36)$.

Tabla 3: Uso principal de la computadora.

\begin{tabular}{|c|c|c|c|}
\hline & & FRECUENCIA & PORCENTAJE \\
\hline \multirow{7}{*}{ Válidos } & Conversación con amigos y familiares & 134 & 30.8 \\
\hline & Correo electrónico & 72 & 16.6 \\
\hline & Redes sociales & 61 & 14.0 \\
\hline & Búsqueda de información & 36 & 8.3 \\
\hline & Descarga de música / videos & 19 & 4.4 \\
\hline & No contestó & 113 & 26.0 \\
\hline & TOTAL & 435 & 100.0 \\
\hline
\end{tabular}

Fuente: Elaboración propia.

La comunicación con los familiares y/o amigos es la razón principal para usar las redes sociales $(42.1 \%, 183)$, seguido del pasatiempo $(26.0 \%, 113)$ y trabajo $(8.7 \%, 38)$. El principal motivo por el que las personas mayores utilizan Internet es la búsqueda de información e indican que lo hacen a menudo $(35.5 \%)$ o mucho $(27.9 \%)$.

Tabla 4: ¿El uso de las redes le proporciona algún sentimiento?

\begin{tabular}{|l|l|r|r|}
\hline \multicolumn{2}{|l|}{} & FRECUENCIA & PORCENTAJE \\
\hline \multirow{4}{*}{ Válidos } & Alegría & 215 & 49.4 \\
\cline { 2 - 4 } & Sorpresa & 78 & 17.9 \\
\cline { 2 - 4 } & Miedo & 34 & 7.8 \\
\cline { 2 - 4 } & No contestó & 108 & 24.8 \\
\cline { 2 - 4 } & TOTAL & $\mathbf{4 3 5}$ & $\mathbf{1 0 0 . 0}$ \\
\hline
\end{tabular}

Fuente: Elaboración propia. 
La tabla 4 nos muestra que el adulto mayor manifiesta que el sentimiento percibido al participar en las redes sociales con sus pares es de alegría $(49.4 \%, 215)$, sorpresa $(17.9 \%$, 78 ) y miedo $(7.8 \%, 34)$. Un $6.5 \%$ (12) respondió que facilita tareas y trabajos, código que significa, que lo hace sentir útil y puede trabajar desde su casa.

Tabla 5: ¿Por qué el uso de las redes le proporciona algún sentimiento?

\begin{tabular}{|c|l|r|r|}
\hline \multicolumn{1}{|c|}{} & FRECUENCIA & PORCENTAJE \\
\hline \multirow{5}{*}{ Válidos } & Sirve para comunicarse con familia, amigos y conocidos & 136 & 31.3 \\
\cline { 2 - 4 } & Sirve para encontrar información & 62 & 14.3 \\
\cline { 2 - 4 } & Le divierte lo que puede ver, oír o hacer & 48 & 11.0 \\
\cline { 2 - 4 } & Miedo al uso de las TIC & 36 & 8.3 \\
\cline { 2 - 4 } & Facilita tareas y trabajos & 21 & 4.8 \\
\cline { 2 - 4 } & No contestó & 132 & 30.3 \\
\cline { 2 - 4 } & TOTAL & 435 & $\mathbf{1 0 0 . 0}$ \\
\hline
\end{tabular}

Fuente: Elaboración propia.

La tabla 5 remarca el sentimiento de alegría como el código más repetido, ya que sirve para comunicarse con familiares, amigos y conocidos $(31.3 \%$, 136), porque les sirve para encontrar información diversa $(14.3 \%, 62)$ y porque se divierten con lo que pueden ver, oír o hacer $(11 \%, 48)$.

Tabla 6: ¿Cuáles son los contenidos que más compartes a través de las redes sociales?

\begin{tabular}{|c|c|c|c|}
\hline & & FRECUENCIA & PORCENTAJE \\
\hline \multirow{9}{*}{ Válidos } & Comunicación & 120 & 27.6 \\
\hline & Información & 83 & 19.1 \\
\hline & Cultura - entretenimiento & 63 & 14.5 \\
\hline & Religión & 27 & 6.2 \\
\hline & Política & 10 & 2.3 \\
\hline & Otros & 10 & 2.3 \\
\hline & Humanismo & 4 & .9 \\
\hline & No contestó & 118 & 27.1 \\
\hline & TOTAL & 435 & 100.0 \\
\hline
\end{tabular}

Fuente: Elaboración propia. 
La tabla 6 deja de manifiesto que los adultos mayores no son sólo receptores de contenidos, sino también generan circulación de información, siendo principalmente esta de carácter comunicativo $(27.6 \%, 120)$, informativo $(19.1 \%, 83)$, cultural y de entretenimiento $(14.5 \%$, $63)$, religioso $(6.2 \%, 27)$, entre otros.

\section{Discusión y conclusiones}

La brecha digital continúa siendo una variable para el empleo de las TIC por las personas mayores de 60 años y que conforme la edad es más avanzada, dicha brecha se acentúa, quizá porque muchos de los dispositivos electrónicos no están adaptados para su empleo por estas personas, quienes (en algunos casos) muestran algún tipo de disminución en sus habilidades o capacidades como la motricidad, la visión o la reacción rápida a estímulos.

Ante los nuevos estilos de vida de las grandes ciudades, resulta básico el empleo de este tipo de tecnologías para mantener una comunicación rápida, práctica y sin costo. Es por ello que los adultos mayores enfilan el uso de los dispositivos para comunicarse con familiares y amistades. La red social más utilizada por los encuestados fue Facebook, lo que confirma que en México es la plataforma digital para compartir información más difundida y empleada. Los adultos mayores usan Facebook para mantenerse al tanto de las actividades de otras personas con las que usualmente tienen una interacción en su vida cotidiana.

Un factor que mantiene esta brecha generacional en los adultos mayores es la falta de capacitación para el empleo de las TIC. Uno de los principios del aprendizaje significativo es motivar el aprendizaje en sí y más cuando se trata de nuevos conocimientos (Hernández \& Hernández, 2014), por lo que las políticas (que beneficien a este colectivo) deberían trazar una estrategia de re-educación, a fin que las TIC en los adultos mayores fomente (aparte del entretenimiento), la formación intelectual y la generación de negocios, tal como lo hacen generaciones más jóvenes.

Al emplear las TIC, los adultos mayores tienen una buena opinión de ellas y las aprovechan. Muñoz (2002) propone que los centros de educación y residencias deberían convertirse en lugares de formación y facilitar su acceso y manejo. Sabemos que en el caso de los dos municipios seleccionados, esto ya se hace, a través de la administración pública. Sin embargo, es necesario extender la red que asegure una cobertura más amplia de este tipo de servicios para mejorar la calidad de vida de los adultos mayores.

La muestra de adultos mayores emplea las TIC, así como el teléfono móvil, para tener conectividad en Internet. Asimismo, la mayoría de esta hace uso diario de estos dispositivos para mantenerse online. La red social más utilizada es el Facebook y es consultada diariamente, al menos, durante una hora. Este consumo está correlacionado consistentemente con la cantidad de dispositivos. Mientras las personas se hacen mayores, van dejando de usar redes sociales, Internet y herramientas digitales. 
En suma, las redes sociales son utilizadas por los adultos mayores porque les genera un sentimiento de alegría: estos recursos les permite divertirse, entretenerse y comunicarse con sus familiares, superando el miedo que les produce oprimir alguna herramienta de su dispositivo electrónico y provocar un problema.

\section{Referencias}

Abad, L. (2014). Diseño de programas de e-inclusión para alfabetización mediática de personas mayores. Comunicar, 42(21), 173-180. doi: 10.3916/C42-2014-17

Agudo, S., Pascual, M. \& Fombona, J. (2012). Usos de las herramientas digitales entre las personas mayores. Comunicar, 39(20), 193-201. doi: 10.3916/C39-2012-03-10

Barroso, J., Cabero, J. \& Romero, R. (2002). Las personas mayores y las nuevas tecnologías: una acción en la sociedad de la información. Innovación educativa, 12, 319-337.

Cardozo, C., Martin, A. \& Saldaño, V. (2017). Los adultos mayores y las redes sociales: Analizando experiencias para mejorar la interacción. Informes Científicos Técnicos UNPA, 9(2), 1-29. doi: 10.22305/ict-unpa.v9i2.244

Cerda, A. (2005). Alfabetización digital en el adulto mayor ¿en el camino de la inclusión social? [tesis inédita de maestría]. Universidad de Chile, Santiago de Chile.

Colombo, F., Aroldi, P. \& Carlo, S. (2015). Nuevos mayores, viejas brechas: TIC, desigualdad y bienestar en la tercera edad en Italia. Comunicar, 45(23), 47-55. doi: http://dx.doi.org/10.3916/C45-2015-05

Culver, S. \& Jackobson, T. (2012). Alfabetización mediática como método para fomentar la participación cívica. Comunicar, 39(29), 73-80. doi: 10.3916/C39-2012-02-07

Cumbre Mundial sobre la Sociedad de la Información (2004). Plan de Acción. Recuperado de https://www.itu.int/dms_pub/itu-s/md/03/wsis/doc/S03-WSISDOC-0005!!PDF-S.pdf

Hernández, H. \& Hernández, M. (2014). El uso de las TIC en la población mayor (tesis de pregrado). Universidad Tecnológica de Pereira. Colombia.

Instituto Nacional de Estadística y Geografía, INEGI (2014). Perfil sociodemográfico de adultos mayores. Recuperado de http://internet.contenidos.inegi.org.mx/ contenidos/productos/prod_serv/contenidos/espanol/bvinegi/productos/censos/ poblacion/2010/perfil_socio/adultos/702825056643.pdf 
Instituto Nacional de Estadística y Geografía, INEGI (2015). Encuesta Intercensal 2015. Recuperado de http://www.beta.inegi.org.mx/proyectos/enchogares/ especiales/intercensal/

Instituto Nacional de las Mujeres de México, Inmujeres (2015). Situación de las personas adultas mayores en México. El arte de envejecer es el arte de conservar alguna esperanza. Recuperado de http://cedoc.inmujeres.gob.mx/documentos download/101243_1.pdf

Kiel, J. (2005). The digital divide: Internet and e-mail use by the elderly. Informatics for Health and Social Care, 30(1), 19-23. doi: 10.1080/14639230500066900

Montaña, M., Estanyol, E. \& Lalueza, F. (2015). Internet y nuevos medios: estudio sobre usos y opiniones de las personas mayores en España. El profesional de la información, 6(24), 759-765.

Muñoz, L. (2002). Las personas mayores ante las tecnologías de la información y la comunicación. Estudio valorativo. Profesorado. Revista de Currículum y Formación del Profesorado, 6(1-2), 1-9. Recuperado de http://www.redalyc.org/ pdf/567/56751267017.pdf

Organisation forEconomic Co-OperationandDevelopment, OCDE(2011). Understanding the Digital Divide. Recuperado de www.oecd.org/dataoecd/38/57/1888451.pdf

Padilla, D. \& Padilla A. (2008). Tecnologías para mayores. Universitas Psychologica, 7(3), 883-894. Recuperado de http://www.redalyc.org/pdf/647/64770320.pdf

Pavón Rabasco, F. (2000). Tecnologías avanzadas: nuevos retos de comunicación para los mayores. Comunicar, 15, 133-139. Recuperado de http://www.redalyc.org/ articulo.oa? $\mathrm{id}=15801521$

Pensionissste (2017). Día del adulto mayor. Recuperado de https://www.gob.mx/ pensionissste/articulos/dia-del-adulto-mayor-123010?idiom=es

Robles, J. \& Molina, O. (2007). La brecha digital: ¿Una consecuencia más de las desigualdades sociales? Un análisis de caso para Andalucía. Empira. Revista de metodología de ciencias sociales, 13, 81-89. Recuperado de http://revistas.uned. es/index.php/empiria/article/view/1160

Vilte, D., Martin A., Gaetan, G. \& Saldaño, V. (2014). Favoreciendo el acceso a la web: experiencias con usuarios mayores de la región patagónica. Recuperado de https://dialnet.unirioja.es/descarga/articulo/5123617.pdf 